\title{
Prenatal anemia and postpartum haemorrhage risk: A systematic review and meta-analysis
}

\author{
Moshood Omotayo ${ }^{1}$, Ajibola Abioye ${ }^{2}$, Moshood Kuyebi $^{3}$, and Ahizechukwu Eke ${ }^{4}$ \\ ${ }^{1}$ Massachusetts General Hospital \\ ${ }^{2}$ Harvard University T H Chan School of Public Health \\ ${ }^{3}$ Lagos State University Teaching Hospital \\ ${ }^{4}$ Johns Hopkins University School of Medicine
}

October 9, 2020

\begin{abstract}
Background: Postpartum hemorrhage ( $\mathrm{PPH})$ is the leading cause of maternal mortality, with loss of uterine tonicity as the main underlying cause. While mechanistic studies suggest that anemia-induced hypoxia reduces uterine tonicity, there is no meta-analysis of relevant clinical studies. Objectives: To conduct a systematic review/meta-analysis synthesizing clinical studies of the relationship between prenatal anemia and PPH. Search Strategy: We used search terms combining hemorrhage and anemia or their derivatives, and searched PUBMED/Medline, EMBASE, Cochrane, PROSPERO and Web of Science. Selection Criteria: Original peer-reviewed articles that measured an indicator of prenatal anemia and postpartum hemorrhage or postpartum hemorrhage-related mortality in humans. Data Collection and Analysis: Our primary analysis used random effects models to synthesize odds-ratios extracted from the studies. Heterogeneity was formally assessed with the Higgins' I2 statistics and explored using meta-regression and subgroup analysis. Main Results: Overall, anemia was not associated with PPH (OR: 1.39; 95\% (CI): $0.64-3.01)$. There was no statistical association with mild (OR=0.60; 95\% CI: $0.31,1.17$, p-value $=0.13)$, or moderate anemia $(\mathrm{OR}=2.09 ; 95 \% \mathrm{CI}: 0.40,11.1, \mathrm{p}$-value $=0.39)$ and the risk of PPH. Our findings suggest that severe prenatal anemia increases postpartum hemorrhage risk $(\mathrm{OR}=3.54 ; 95 \% \mathrm{CI}: 1.20,10.4$, p-value $=0.02)$. Conclusions: Mild and moderate prenatal anemia is not associated with PPH risk. High quality prospective studies are critical to evaluating the effect of severe anemia on PPH risk and related mortality. Funding: None. Key words: Maternal anemia, Post-partum hemorrhage risk factors, Post-partum hemorrhage-related mortality, Maternal mortality, Obstetric Emergency.
\end{abstract}

\section{Hosted file}

BJOG_Manuscript 10.pdf available at https://authorea.com/users/365882/articles/485813prenatal-anemia-and-postpartum-haemorrhage-risk-a-systematic-review-and-meta-analysis

\section{Hosted file}

BJOG3_Table 1.pdf available at https://authorea.com/users/365882/articles/485813-prenatalanemia-and-postpartum-haemorrhage-risk-a-systematic-review-and-meta-analysis 


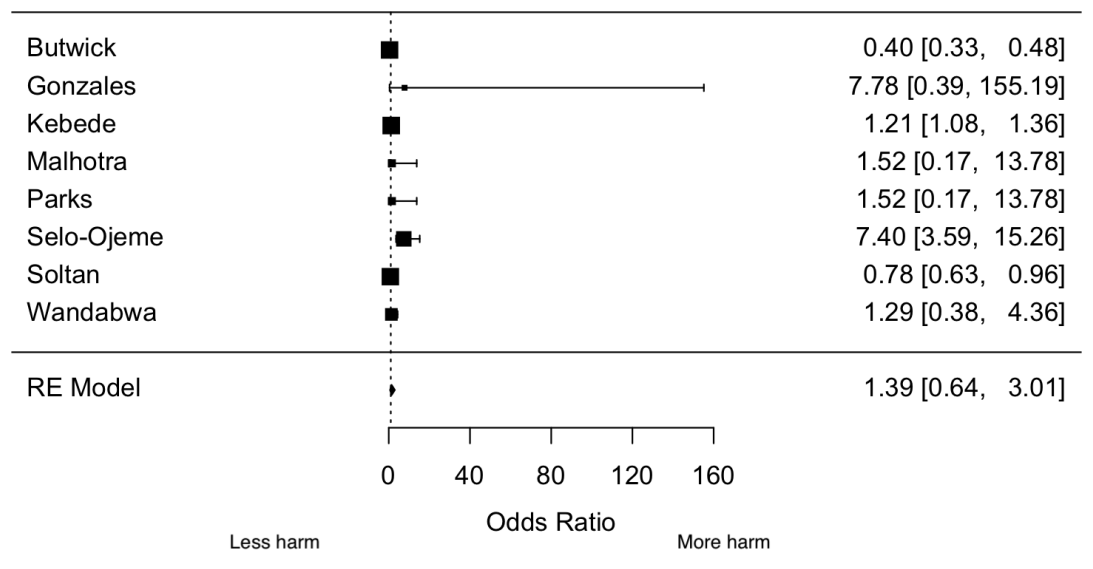

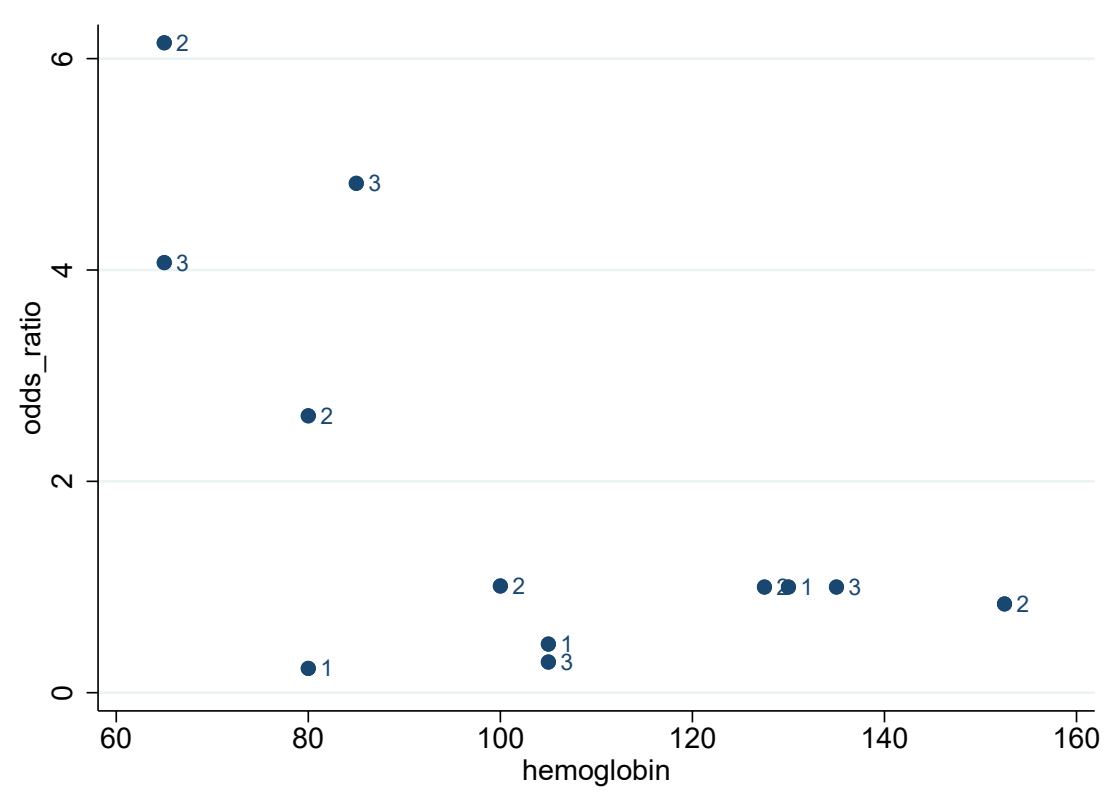




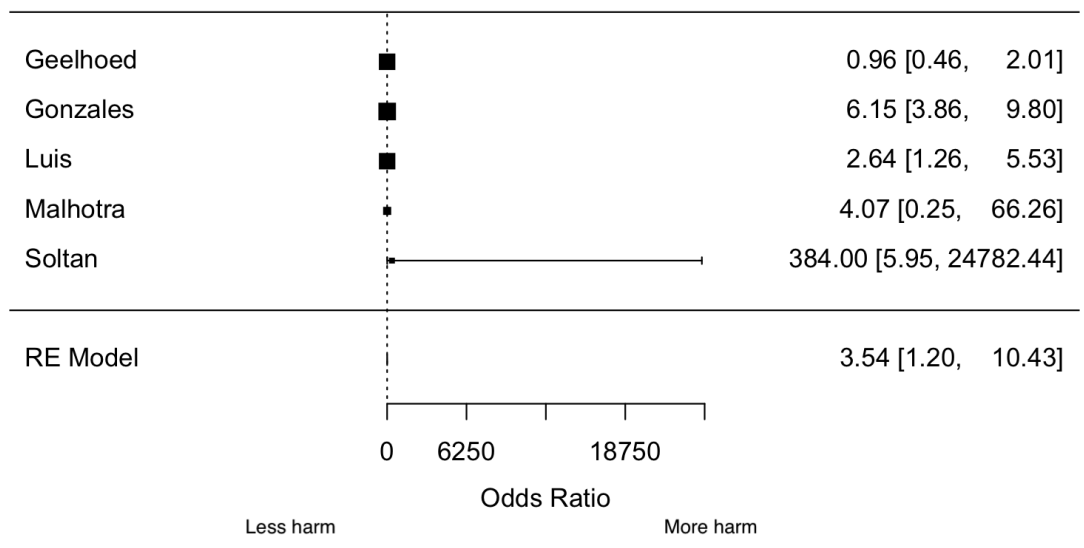

